\title{
Prediction Intervals for the Failure Time of Prestressed Concrete Beams
}

\author{
Sebastian Szugat, Jens Heinrich, Reinhard Maurer, and Christine H. Müller
}

TU Dortmund University, 44227 Dortmund, Germany

Correspondence should be addressed to Sebastian Szugat; szugat@statistik.tu-dortmund.de

Received 24 March 2016; Accepted 20 July 2016

Academic Editor: Konstantinos I. Tserpes

Copyright (C) 2016 Sebastian Szugat et al. This is an open access article distributed under the Creative Commons Attribution License, which permits unrestricted use, distribution, and reproduction in any medium, provided the original work is properly cited.

\begin{abstract}
The aim is the prediction of the failure time of prestressed concrete beams under low cyclic load. Since the experiments last long for low load, accelerated failure tests with higher load are conducted. However, the accelerated tests are expensive so that only few tests are available. To obtain a more precise failure time prediction, the additional information of time points of breakage of tension wires is used. These breakage time points are modeled by a nonlinear birth process. This allows not only point prediction of a critical number of broken tension wires but also prediction intervals which express the uncertainty of the prediction.
\end{abstract}

\section{Introduction}

Actually, the assessment of existing prestressed concrete bridges by means of recalculation in conjunction with rehabilitation and strengthening is gaining more and more importance compared to the construction of new bridges. The current design codes had been developed over decades always adapting new design approaches that are current at that time. Even for this reason the recalculation of older existing structures often leads to deficiencies concerning load-bearing capacity, durability, and resistance against fatigue. The ongoing increase of traffic concerning heavy trucks underlines the importance of assessment and maintenance of the transport networks and particularly the bridge stock, the latter with regard to structural safety.

Beside corrosion effects, the major influence for time dependent losses of load-bearing capacity is the phenomenon of fatigue failure. Fatigue is caused by frequent cyclic loads due to the crossing of heavy trucks on the bridge deck. Beside steel bridges, prestressed concrete bridges are affected as well; see, for example, $[1,2]$. For the design of new bridges against fatigue or the assessment of existing bridges by means of recalculation, $S-N$ curves are needed. The latter describes the fatigue resistance of the materials. With regard to the prestressed concrete bridges, this refers especially to the embedded reinforcing and prestressing steel in cracked sections. For the design and assessment of bridges, $S-N$ curves are needed in a range up to $10^{8}$ load cycles. To obtain values for the whole range and for a better understanding of the fatigue behavior of prestressed concrete bridges, one has to carry out long running tests which are extremely expensive. Hence, there is a great need to optimize these tests procedures.

From historical view, the first documented fatigue tests on prestressed concrete beams will be found in [3]. Larger test series carried out at the University of Texas are described by [4]. Further studies can be found in [5, 6]. A comprehensive survey regarding fatigue tests on prestressing steel in air and embedded in concrete is given in [7]. The latter leads to fatigue strength which is significantly less than studied before.

During the course of the Collaborative Research Center SFB 823 Statistical Modeling of Nonlinear Dynamic Processes, large-scale test series with stress ranges down to $50 \mathrm{MPa}$ and failure times in a range up to $10^{8}$ load cycles are carried out at TU Dortmund University. The aim of the ongoing experimental studies described subsequently is to investigate fatigue behavior and to provide characteristic $S-N$ curves for prestressing steel in curved steel ducts embedded 
in concrete of posttensioned members. $S-N$ curves belong to the basics which are needed to verify prestressed concrete bridges against fatigue. However, tests under cyclic loading of posttensioned concrete beams may be very time-consuming and expensive. Especially at very low stress ranges with a very high number of cycles, which are of particular interest concerning prestressed concrete bridges, even an optimized test with a realized load frequency of $10 \mathrm{~Hz}$ lasts several months. For posttensioned steel, an endurance range in the $S-N$ curves has not been established by tests up to now. Therefore, the $S-N$ curve in the range up to $10^{8}$ cycles can only hypothetically be guessed, until appropriate test results will be available.

For low loads down to $60 \mathrm{MPa}$, tests in the research project SFB 823 last nearly 100 days so that most experiments are done under higher loads up to $200 \mathrm{MPa}$. Hence so-called accelerated failure tests (AFT) were conducted. If there are enough AFT experiments, the lifetime at a small load can be estimated from the $S-N$ curves; see, for example, [8-10]. However, here also these AFT experiments last long and are expensive so that the results of only few experiments are available, in our project, for example, results of ten experiments. Such small numbers of experiments are too small to estimate the lifetime at low load with enough precision. Nevertheless, the main interest lies in the lifetime at low stress at $50 \mathrm{MPa}$ or even lower.

Hence, we propose here two methods which use additional information beside the failure times of former tests to predict the failure time at low stress. The additional information is given by a degradation measure. Usually the sizes of cracks are used as degradation measures; see, for example, [11-13]. But here we have the advantage that the time points of the breaking of the tension wires in the prestressed concrete beams are available since acoustic signals obtained by a microphone indicate clearly the breakage of a wire. We model the time points of the breaking of the tension wires with a point process where the waiting times for the next breaking of a tension wire follow an exponential distribution depending only on the number of wires which are broken before. Such point processes are also called birth processes (see, e.g., [14]).

Point processes as Poisson processes and renewal processes are often considered in reliability and lifetime analysis; see, for example, $[15,16]$. Reference [15] treats also a linear birth process for fatigue accumulation in Chapter 18 and uses birth processes with time-varying intensity for crack growth in Chapter 26. However, our birth process is nonlinear in the number of broken tension wires. The nonlinearity is due to the redistribution of the load on the tension wires. There are several approaches for load sharing systems as those of $[17,18]$ or [19]. But they assume several systems exposed to the same stress so that accelerated failure tests cannot be treated.

Linking the nonlinear birth process of each experiment with its underling stress, we provide two types of prediction intervals for the time of a critical number of broken tension wires. The critical number of broken tension wires has a direct relation to the failure time of the concrete beam so that its lifetime can be derived from the time of the critical number of broken tension wires. We use the times between successive breaks of the accelerated experiments and some optional first breaking times of the concrete beam for which we want to obtain the prediction interval.

Although prediction intervals provide not only a prediction but also its precision, they are often not derived. Most prediction intervals are only derived for the simple situation that all experiments are conducted under the same conditions; see, for example, [8, 9, 20-25]. Only few prediction intervals for accelerated experiments are available as those of [8] for normal distributed lifetimes and [26] for exponential distributed lifetimes while the prediction intervals of [26] are based on simulations. Our prediction intervals are simulation-free and thus faster to calculate.

In Section 2 the description of the experiments with the concrete beams is given. Section 3 provides the statistical model with the birth process and its link to the stress while Section 4 treats the two proposed prediction intervals. The results for our experiments with the concrete beams and some simulations are given in Section 5. At last, Section 6 provides a conclusion.

\section{Test Setup and Procedure}

The tests on prestressed concrete girders (hereinafter SB01SB05) within the Collaborative Research Center SFB 823 have been carried out at TU Dortmund University. The experimental setup is based on the setup of already conducted experiments, also carried out at TU Dortmund University (see [27]).

The series described in [27] consisted of five concrete girders (TR01-TR05) with tendons for posttensioning. They had been tested with different stress ranges $\Delta \sigma_{p}$ from $455 \mathrm{MPa}$ to $98 \mathrm{MPa}$ for the prestressing steel in curved steel ducts. The prestressing steel of the tendons used for these test girders has been taken from an existing bridge which was built in 1957 and demolished in 2007. Each of the taken $3 / 8^{\prime \prime}$ strands consists of seven single wires. Each strand had been consisted of a steel grade St1570/1770 with a diameter of $9.3 \mathrm{~mm}$ and a cross-sectional area of $52 \mathrm{~mm}^{2}$. The prestressing steel had been strained at a length of $2 \mathrm{~m}$ for the curved tendon with a minimum radius of $r=5 \mathrm{~m}$ in a region of the test girder with pure bending without shear. Hence, the influence of fretting corrosion between the tensioned strand and steel duct is included.

The experimental setup consists of steel frames, the concrete girder, and a hydraulic press in a four-column testing machine, which can apply a cyclic load at maximum $\pm 2500 \mathrm{kN}$ (see Figures 1 and 2 ). The overall dimensions of the concrete girder are $4.00 \mathrm{~m} \times 1.00 \mathrm{~m} \times 0.30 \mathrm{~m}$. A recess in midspan of the girder in conjunction with a steel contact element ensures the unambiguous definition of the center of the compression zone in the upper cross-sectional part and from this the exact inner lever arm and tension force in the tendon.

The test girders of the second test series SB01-SB05 are very similar to those of the first test series TR01-TR05. A few modifications like a steel-link in the pressure area in the girder's center and the prestressing of the anchoring rods increase the stiffness of the whole test stand and the test 


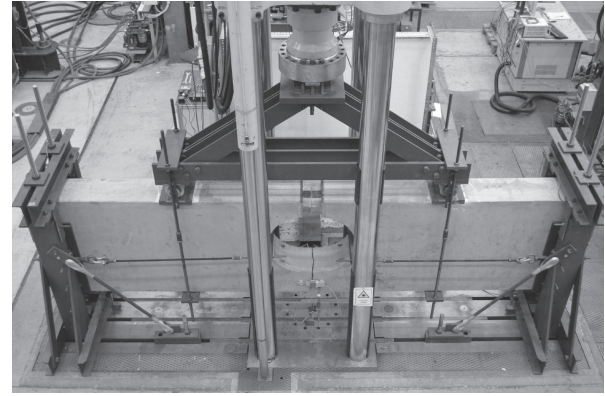

FIGURE 1: Photograph of the experimental setup.

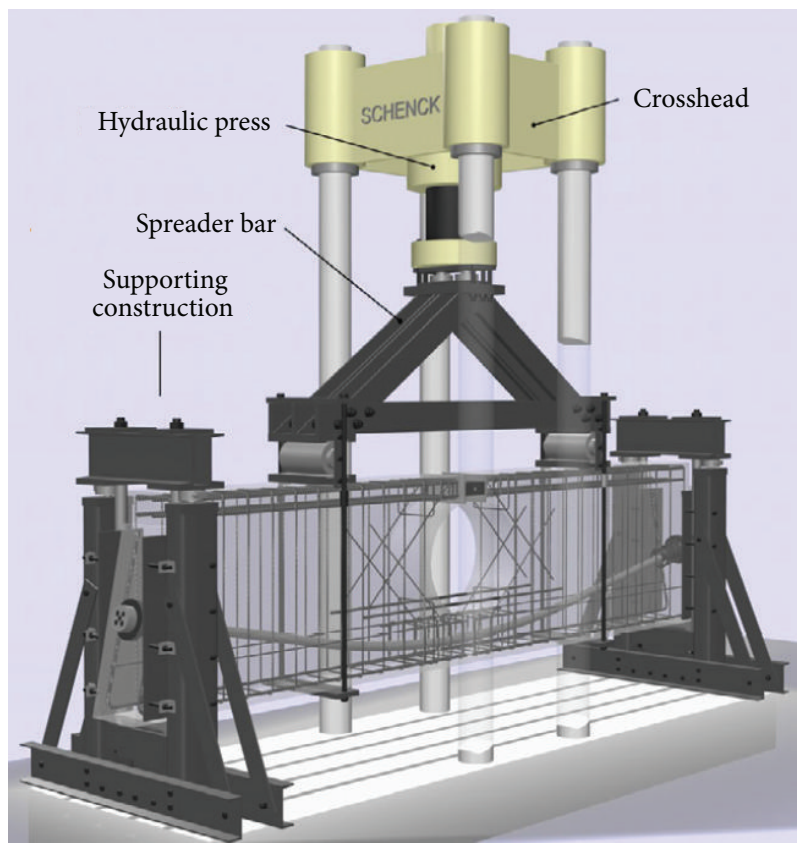

FIGURE 2: 3D CAD model of the test stand.

frequency, whereby the duration could be reduced. The test frequency was set at $1.5-2 \mathrm{~Hz}$ for the first test series and was optimized up to $10 \mathrm{~Hz}$ for the second test series.

The experimental procedure was the same for both test series and will be described below. Firstly, all the press force was applied to the concrete girder. The load was increased continuously until an initial crack in the tension zone appears and a bearing effect of the concrete in tension could be excluded. Initially the girder has been released in a way, so that the load could be increased up to the respective medium load range. After that, the fatigue strength of the embedded prestressing steel was tested under a constant cyclic loading until a critical number of the wires had broken due to fatigue and the remaining section could no longer withstand the load.

During the experiment runtime, the crack width in midspan of the girder was measured continuously. As soon as a wire has broken due to fatigue, the measurement of the crack width showed a sudden increase. The amount of increase depends on the total number of already broken

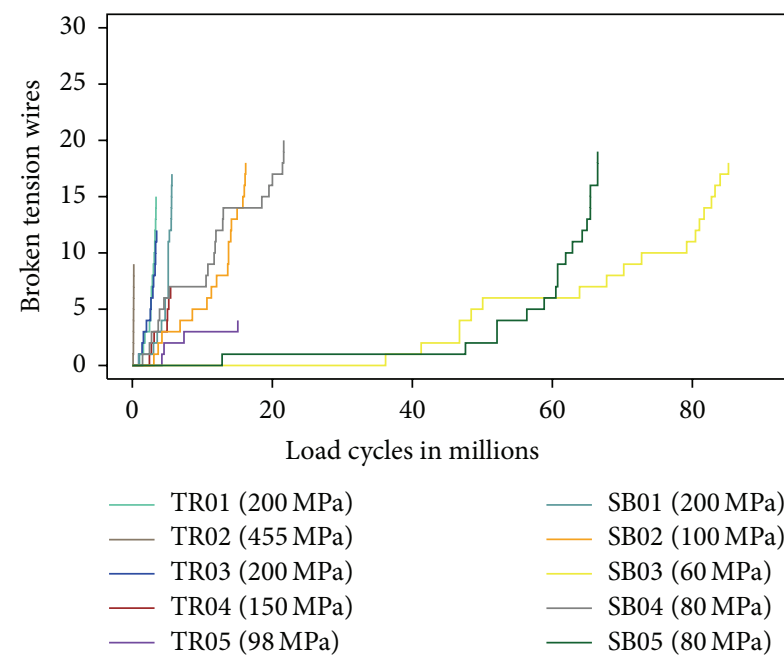

FIGURE 3: Tests results for TR01-TR05 and SB01-SB05.

wires. The more the wires were broken, the greater the sudden increase was (see [7]). Although the time point of the breaking of a tension wire could be determined by the sudden increase of the crack width, a more precise determination of the breaking times was obtained with a microphone since each breaking causes a short load noise.

It could be that more than one tension wire is breaking at a time point. However, this cannot be determined, neither by the crack width nor by microphone. But this should be a rare event so that we neglect this possibility in our model.

The applied stress range $\Delta \sigma_{p}$ and the time of every broken wire in each of test girders are shown in Figure 3.

\section{Statistical Model}

Let $n$ be the time measured in load cycles and $\mathcal{N}_{n}$ the number of broken prestressing wires up to time $n$. The waiting time between the $i-1^{\prime}$ th and the $i^{\prime}$ th broken wire is defined as $\Delta N_{i}:=\min \left\{n: \mathcal{N}_{n} \geq i\right\}-\min \left\{n: \mathcal{N}_{t} \geq i-1\right\}$. The exponential distribution is commonly used to model lifetimes. Hence, we assume that

$$
\Delta N_{i} \sim \operatorname{Exp}\left(\lambda_{\theta}(i-1, s)\right), \quad i=1, \ldots, I \leq I_{\max },
$$

where $\lambda_{\theta}(i-1, s)$ is the parameter of the exponential distribution, which depends on the number of broken wires $i$ and the stress range $s:=\Delta \sigma_{p}$ of the experiment. $I_{\max }$ denotes the maximum number of possible wire breakages, so that we have $I_{\max }=35$ here, because there are five strands with seven wires each embedded in a beam.

Note that $\mathcal{N}_{n}$ is the classical Poisson process if $\lambda_{\theta}$ does not depend on the number $i$ of broken tension wires. If it depends on $i$, it is birth process; see, for example, [14].

A simple assumption for $\lambda_{\theta}(i-1, s)$ in the experiments with prestressed concrete is

$$
\lambda_{\theta}(i, s):=h_{\theta}\left(s \cdot \frac{I_{\max }}{I_{\max }-i}\right)
$$


for a function $h_{\theta}$ which depends on $\theta \in \Theta$. The term $I_{\max } /\left(I_{\max }-i\right)$ expresses the increase of stress on the remaining $I_{\max }-i$ wires when $i$ wires are broken. In particular, when half of the wires are broken $\left(i=I_{\max } / 2\right)$ then the stress is doubled. The function $h_{\theta}$ models the dependence of the waiting time for the next breakage on the stress of the remaining tension wires. In this work we choose

$$
h_{\theta}(x):=\exp \left(-\theta_{1}+\theta_{2} \log (x)\right)
$$

with $\theta=\left(\theta_{1}, \theta_{2}\right)^{\top} \in \Theta=[0, \infty)^{2}$ so that

$$
\begin{aligned}
\log \left(E\left(\Delta N_{i}\right)\right) & =\log \left(\frac{1}{\lambda_{\theta}(i-1, s)}\right) \\
& =\theta_{1}-\theta_{2} \cdot \log \left(s \cdot \frac{I_{\max }}{I_{\max }-i+1}\right) ;
\end{aligned}
$$

that is, it is assumed that the expected time until the next wire break can be modeled in that way. This model for the logarithmized expectation coincides with a well-known and used model in the engineering sciences from [28].

Since we do not only have one experiment with one stress level $s$ but also have $J$ experiments with different stress ranges $s_{1}, \ldots, s_{J}$, we observe realizations $\Delta n_{i, j}$ of

$$
\Delta N_{i, j} \sim \operatorname{Exp}\left(\lambda_{\theta}\left(i-1, s_{j}\right)\right)
$$

for $i=1, \ldots, I_{j} \leq I_{\max }, j=1, \ldots, J$.

For the prediction we have a new beam experiment with realizations $\Delta n_{i, 0}$ of

$$
\Delta N_{i, 0} \sim \operatorname{Exp}\left(\lambda_{\theta}\left(i-1, s_{0}\right)\right)
$$

with $i=1, \ldots, I_{0} \ll I_{\max }$. We use $I_{0}=0$ if no observations of broken tension wires are available for this experiment. In particular, our aim is also to make predictions for low stress levels where no experiments were conducted up to now.

Let $I_{\text {crit }}$ denote a critical number of broken tension wires which is closely related to the lifetime of the concrete beam. Then we want to predict the time of the $I_{\text {crit }}$ 'th failure $\left(I_{0}<\right.$ $\left.I_{\text {crit }}<I_{\max }\right)$, that is, the time measured in load cycles given by

$$
\Delta n_{1,0}+\cdots+\Delta n_{I_{0}, 0}+\Delta N_{I_{0}+1,0}+\cdots+\Delta N_{I_{\text {crit }}, 0} .
$$

Since $\Delta n_{1,0}, \ldots, \Delta n_{I_{0}, 0}$ have already been observed, the task reduces to the prediction of the future sum of waiting times:

$$
\Delta N_{\text {fut }}:=\Delta N_{I_{0}+1,0}+\cdots+\Delta N_{I_{\text {crit }}, 0} .
$$

\section{Prediction Intervals}

If the parameter $\theta=\left(\theta_{1}, \theta_{2}\right)^{T}$ is known, then the prediction for the expected time (number of load cycles) until the number of broken tension wires attains the critical number $I_{\text {crit }}$ is

$$
\begin{aligned}
& \Delta n_{1,0}+\cdots+\Delta n_{I_{0}, 0}+\frac{1}{\lambda_{\theta}\left(I_{0}, s_{0}\right)}+\cdots \\
& +\frac{1}{\lambda_{\theta}\left(I_{\text {crit }}-1, s_{0}\right)}
\end{aligned}
$$

since the expectation of random variable $\Delta N_{i, 0}$ with exponential distribution satisfies $E\left(\Delta N_{i, 0}\right)=1 / \lambda_{\theta}\left(i-1, s_{0}\right)$.

However, such a point prediction will usually fail the true future time. To include the precision of the prediction, a prediction interval for $\Delta N_{\text {fut }}$ and thus for $\Delta n_{1,0}+\cdots+\Delta n_{I_{0}, 0}+$ $\Delta N_{\text {fut }}$ is needed. A $(1-\alpha)$-prediction interval $\mathbb{P}$ for the future value of $\Delta N_{\text {fut }}$ should satisfy

$$
P\left(\Delta N_{\text {fut }} \in \mathbb{P}\right) \geq 1-\alpha,
$$

where $\alpha$ is usually a small value like $\alpha=0.1$. It means that the future observation $\Delta N_{\text {fut }}$ lies in the prediction interval $\mathbb{P}$ with a probability greater than $1-\alpha$, for example, $90 \%$ if $\alpha=0.1$. The smaller $\alpha$ is and thus the larger $1-\alpha$ is, the larger and more noninformative the prediction interval is. Therefore $\alpha=0.1$ is a good choice since the probability is at least $90 \%$ that the prediction interval includes the future observation.

In order to find a prediction interval for $\Delta N_{\text {fut }}$, the distribution of $\Delta N_{\text {fut }}$ in expression (8) is needed. As $\Delta N_{\text {fut }}$ is the sum of exponential distributions each with a different parameter, it is hypoexponentially distributed (see, e.g., [29, pp. 293]) with cummulative distribution function:

$$
\begin{aligned}
& F_{\Delta N_{\text {fut }}, \theta}\left(\Delta n_{\text {fut }}\right) \\
&:=\sum_{i=I_{0}+1}^{I_{\text {crit }}} a_{i}(\theta)\left(1-\exp \left(-\Delta n \lambda_{\theta}\left(i-i, s_{0}\right)\right)\right),
\end{aligned}
$$

where $a_{i}(\theta):=\prod_{k=I_{0}+1, k \neq i}^{I_{\text {crit }}}\left(\lambda_{\theta}\left(k, s_{0}\right) /\left(\lambda_{\theta}\left(k, s_{0}\right)-\lambda_{\theta}\left(i, s_{0}\right)\right)\right)$. Expression (11) can be numerically instable if it is implemented directly and $I_{\text {crit }}-I_{0}$ is large or the parameters $\lambda_{\theta}$ of the single exponential distributions do not differ much. In this case [30] provides a more stable implementation based on the matrix exponential (see [31]).

An $\alpha$-quantile $b_{\alpha}(\theta)$ of the hypoexponential distribution can be computed implicitly by solving

$$
F_{\Delta N_{\text {fut }}, \theta}\left(b_{\alpha}(\theta)\right)-\alpha=0 .
$$

Hence, if the parameter $\theta$ is known then

$$
\mathbb{P}:=\left[b_{\alpha / 2}(\theta), b_{1-\alpha / 2}(\theta)\right]
$$

is a prediction interval for $\Delta N_{\text {fut }}$ since $P\left(\Delta N_{\text {fut }} \in \mathbb{P}\right)=1-\alpha$.

However, the parameter $\theta=\left(\theta_{1}, \theta_{2}\right)^{T}$ is not known in practice and has to be estimated from the data $\Delta n_{\text {all }}:=$ $\left(\Delta n_{1,0}, \ldots, \Delta n_{I_{0}, 0}, \ldots, \Delta n_{1, J}, \ldots, \Delta n_{I_{I}, J}\right)$ given by the experiments. A commonly used estimator for $\theta$ is the maximum likelihood estimator:

$$
\widehat{\theta} \in \underset{\theta \in \Theta}{\operatorname{argmax}} f_{\theta, \text { all }}\left(\Delta n_{\text {all }}\right),
$$

where

$$
f_{\theta, \text { all }}\left(\Delta n_{\text {all }}\right):=\prod_{j=0}^{J} \prod_{i=1}^{I_{j}} f_{\lambda_{\theta}\left(i, s_{j}\right)}\left(\Delta n_{i, j}\right)
$$

is the density of the distribution of all data and $f_{\lambda}(\Delta n):=\lambda \exp (-\lambda \Delta n)$ is the density of the exponential 
distribution. The density $f_{\theta \text {,all }}\left(\Delta n_{\text {all }}\right)$ is given as the product of the single densities $f_{\lambda_{\theta}\left(i, s_{j}\right)}\left(\Delta n_{i, j}\right)$ because $\Delta N_{1,0}, \ldots, \Delta N_{I_{0}, 0}, \ldots, \Delta N_{1, J}, \ldots, \Delta N_{I_{I}, J}$ are stochastically independent.

Having an estimator $\widehat{\theta}$ for $\theta$, the prediction of the time of the critical number $I_{\text {crit }}$ of broken tension wires is

$$
\begin{aligned}
& \Delta n_{1,0}+\cdots+\Delta n_{I_{0}, 0}+\frac{1}{\lambda_{\hat{\theta}}\left(I_{0}, s_{0}\right)}+\cdots \\
& +\frac{1}{\lambda_{\hat{\theta}}\left(I_{\text {crit }}-1, s_{0}\right)} .
\end{aligned}
$$

If $\theta$ is unknown, then the prediction interval for the future $\Delta N_{\text {fut }}$ must depend on the available data $\Delta n_{\text {all }}$ which is a realization of the random vector $\Delta N_{\text {all }}$. Hence, it is a function $\mathbb{P}\left(\Delta n_{\text {all }}\right)$ of $\Delta n_{\text {all }}$ and it is an exact prediction interval if $P_{\theta}\left(\Delta N_{\text {fut }} \in \mathbb{P}\left(\Delta N_{\text {all }}\right)\right) \geq 1-\alpha$ for all possible $\theta$. That means for different realizations $\Delta n_{\text {all }}$ we get different prediction intervals. A naive prediction interval for $\Delta N_{\text {fut }}$ is given by (see [9])

$$
\mathbb{P}:=\left[b_{\alpha / 2}(\widehat{\theta}), b_{1-\alpha / 2}(\widehat{\theta})\right],
$$

where $\widehat{\theta}$ is the maximum likelihood estimator. This is an approximate $(1-\alpha)$-prediction interval only for large sample sizes, because it does not include any information about the uncertainty of the estimation. For a small number of observations the coverage probability may differ drastically from $1-\alpha$ (see $[9$, p. 294]). We will analyze this in a simulation study in Section 5.

To include the uncertainty of the estimator $\widehat{\theta}$, a confidence interval for $\theta$ can be used. A $(1-\alpha)$-confidence interval $\mathbb{C}$ for $\theta$ depends also on the available observation vector $\Delta n_{\text {all }}$ and satisfies $P\left(\theta \in \mathbb{C}\left(\Delta N_{\text {all }}\right)\right) \geq 1-\alpha$ for all $\theta \in \Theta$. A $(1-\alpha)$ confidence set for $\theta$ can be derived using a likelihood ratio test (see, e.g., [32, pp. 409]). It is given by

$$
\widehat{\mathbb{C}}:=\left\{\theta:-2 \log \left(\frac{f_{\theta, \text { all }}\left(\Delta n_{\text {all }}\right)}{f_{\widehat{\theta}, \text { all }}\left(\Delta n_{\text {all }}\right)}\right) \leq \chi_{2,1-\alpha}^{2}\right\},
$$

if $\chi_{2,1-\alpha}^{2}$ is the $(1-\alpha)$-quantile of the $\chi^{2}$ distribution with 2 degrees of freedom, because we consider $\theta=\left(\theta_{1}, \theta_{2}\right)$; that is, two parameters have to be estimated.

It is possible to use the $(1-\alpha)$-confidence set $\widehat{\mathbb{C}}$ in expression (18) to include the uncertainty of the estimation. A $(1-2 \alpha)$-prediction interval which is also valid for smaller sample sizes is then given by

$$
\begin{aligned}
\mathbb{P} & :=\bigcup_{\theta \in \widehat{\mathbb{C}}}\left[b_{\alpha / 2}(\theta), b_{1-\alpha / 2}(\theta)\right] \\
& \subset\left[\min _{\theta \in \widetilde{\mathbb{C}}} b_{\alpha / 2}(\theta), \max _{\theta \in \widetilde{\mathbb{C}}} b_{1-\alpha / 2}(\theta)\right] ;
\end{aligned}
$$

that is, for all $\theta \in \widehat{\mathbb{C}}$ the corresponding quantiles of the hypoexponential distribution are computed. The minimum over all lower quantiles $b_{\alpha / 2}$ and the maximum over all upper quantiles $b_{1-\alpha / 2}$ are then the desired $1-2 \alpha$ prediction interval for $\Delta N_{\text {fut }}$.

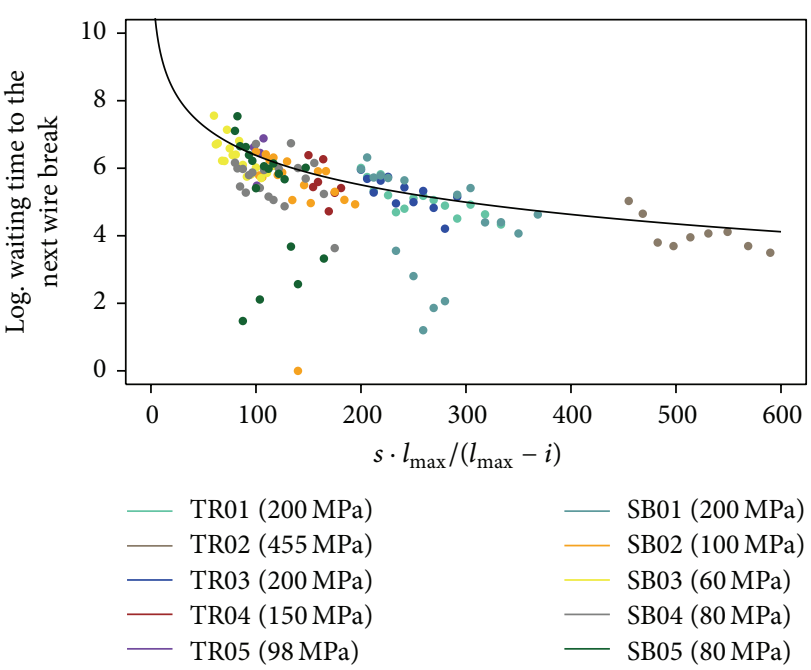

FIGURE 4: Fitted expectation of the logarithmized waiting times using the Basquin link function.

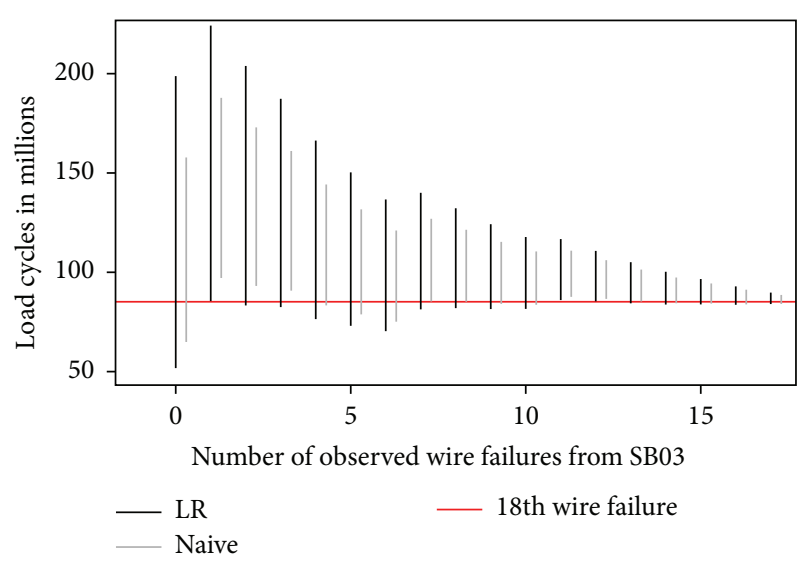

FIGURE 5: 90\% prediction intervals based on the likelihood ratio and the naive method for the 18th wire failure of SB03 with different number of used previous failures from the same beam and all data from the other nine experiments.

\section{Results}

We now apply the proposed methods to the data from the ten experiments described in Section 2. Figure 4 shows the fitted expectation of the logarithmized waiting times when the Basquin link function from Section 3 is used. The relation between the increasing stress on the gradually breaking wires and the waiting time is adequately modeled by this function as it tends to infinity for a stress near 0 and fits the majority of observed waiting times. For the comparison of the two prediction methods, we use the beam SB03 as an example, because with an initial stress range of $60 \mathrm{MPa}$ it is the most interesting one for real applications. In this experiment 18 wire breaks could be observed until the complete failure of the beam. The time of this 18th failure can be predicted if the corresponding observation is removed from the dataset.

In Figure 5, 90\% prediction intervals for this last wire break are shown where a different number of previous broken 


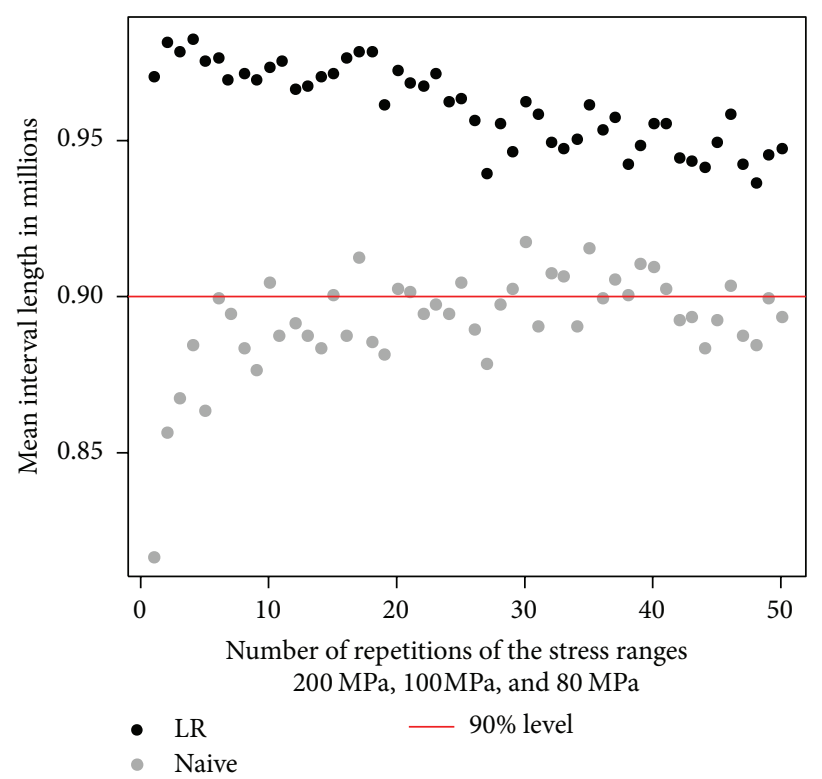

(a)

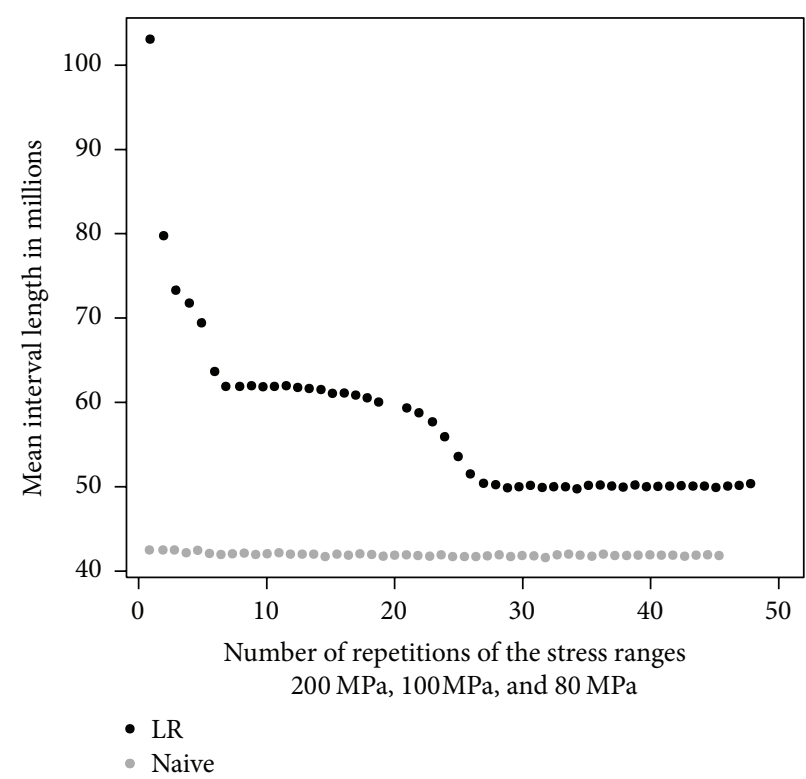

(b)

Figure 6: Coverage rates (a) and mean interval lengths (b) of the $90 \%$ prediction intervals using the likelihood ratio and the naive approach.

wires from the same experiment and all observations from the other experiments are used. The fewer observations from SB03 are used, the more wire failures have to be predicted. Hence, the prediction intervals are larger and get smaller when less breakages are predicted. The naive intervals are always smaller than the ones based on the likelihood ratio approach but the true time of the beam's complete failure is not always covered by the naive intervals, where this is only once the case for the likelihood ratio method. It is obvious that the prediction task is simpler when less wire breaks have to be predicted.

To further check the performance of the two proposed prediction methods, we consider a simulation study. For this, we consider three experiments with initial stress ranges $s_{1}=$ $200 \mathrm{MPa}, s_{2}=100 \mathrm{MPa}$, and $s_{3}=80 \mathrm{MPa}$. Six wire breaks are simulated for each of the three experiments by using the Basquin link function:

$$
\lambda_{\theta}(i, s):=\exp \left(-\theta_{1}+\theta_{2} \log \left(s \cdot \frac{I_{\max }}{I_{\max }-i}\right)\right)
$$

with $I_{\max }=35$ and $\theta=(28.163551,2.922285)^{\top}$, which is the maximum likelihood estimator from the real data of the SFB project.

Furthermore, we generate three wire failures of an additional experiment with $s_{0}=60 \mathrm{MPa}$. For this additional beam, the time of the sixth wire break is predicted using only the first three failure times. This is done with $90 \%$ prediction intervals via the naive and the likelihood ratio approach. Hence, in this first scenario there are $n_{1}=21$ observations to estimate the parameter $\theta$ and to compute the $95 \%$ confidence set based on the likelihood ratio test. The sample size is then subsequently increased by sampling repetitions of the first three experiments, so that all scenarios have $J_{l}=l \cdot 18+3$ observations for $l=1, \ldots, 50$. This results in a maximum considered sample size of $J_{50}=903$.

For each scenario, we check if the simulated time of the sixth breakage which has been removed before the estimation is covered by the two prediction intervals and compare the lengths of the intervals. This procedure is replicated 1000 times for each scenario to get meaningful estimations of the coverage rates and the interval lengths.

Figure 6(a) shows the coverage rate over the 1000 replications. It can be seen that for small sample sizes the naive method does not provide a valid prediction interval as the coverage rate is much lower than $90 \%$. With increasing sample size, the coverage rate is converging to $90 \%$ though. The likelihood ratio approach leads to valid prediction intervals even for a very small number of observations but the resulting intervals are conservative and tend towards a 95\% coverage rate instead of $90 \%$. The intervals based on the confidence sets are always larger than for the naive method because it uses the quantiles $b_{0.025}(\tilde{\theta})$ and $b_{0.975}(\tilde{\theta})$ with $\tilde{\theta} \in \widehat{\mathbb{C}}$, whereas the naive interval uses the smaller quantiles $b_{0.05}(\widehat{\theta})$ and $b_{0.95}(\widehat{\theta})$ based on the maximum likelihood estimator $\hat{\theta}$. With increasing sample size, the $(1-\alpha)$-confidence set $\widehat{\mathbb{C}}$ becomes smaller until it only contains the maximum likelihood estimator. In this case the $90 \%$ interval based on the likelihood ratio confidence set coincides with the naive 95\% prediction interval.

The average lengths of the 1000 prediction intervals for all considered scenarios are depicted in Figure 6(b). For small sample sizes the prediction intervals based on the confidence sets are much larger than the naive ones but they get smaller when the number of observations is increased. In Scenario $i=27$ with 489 observations and all following ones the confidence sets only consist of the maximum likelihood estimator. Hence, the average length of the intervals cannot 
decrease further. Since the naive prediction interval only depends on the maximum likelihood estimator, the average lengths do not vary much for this method in the simulation study.

Summarizing the results of the simulation study, it was shown that for small sample sizes the naive method leads to invalid prediction intervals with too low coverage rates. In this situation the approach based on confidence sets using likelihood ratio tests can be used. For moderate and large samples, the naive prediction intervals are valid though. The prediction intervals based on the confidence sets tend to be conservative as their coverage rate was always above the chosen level of $90 \%$. However, in praxis there oftentimes are only a few experiments due to the immense costs in time and material, so that the confidence set based method is nevertheless a plausible choice.

\section{Conclusion}

The two proposed methods for predicting the failure time of prestressed concrete beams are based on predicting the time when a critical number of tension wires is broken. One method is a naive method using only the maximum likelihood estimator. The other method uses confidence sets given by the likelihood ratio test. Both methods are based on the waitings times between successive breakages of wires. This is possible since the time points can be measured quite precisely with a microphone. Using all available waiting times increases the number of observations substantially which is in particular important when only few experiments with concrete beams can be conducted. Since the number of observations is increased, reasonable prediction intervals can be derived which provide the uncertainty of the prediction. Although a quite simple model of the dependence of the waiting times on the number of tension wires is used, it is shown that both methods provide reasonable results using ten experiments with concrete beams. A simulation study however shows that the naive method should be used with caution if the number of observations is low. The simple model which was used does not take into account any damage accumulation. To include damage accumulation, the waiting times should depend on the waitings times observed before and should have a Weibull distribution with increasing hazard rate. However, it is up to now unclear how to get the estimators, predictors, and prediction intervals then, since the independence of the waiting times is not satisfied anymore.

\section{Competing Interests}

The authors declare that there are no competing interests regarding the publication of this paper.

\section{Acknowledgments}

The research was supported by the Collaborative Research Center SFB 823 Statistical Modeling of Nonlinear Dynamic Processes.

\section{References}

[1] R. Z. Al-Zaid and A. S. Nowak, "Fatigue strength of prestressed concrete girder bridges," Canadian Journal of Civil Engineering, vol. 15, no. 2, pp. 199-205, 1988.

[2] C. Higgins, W. C. Farrow III, B. S. Nicholas, and T. Potisuk, "High-cycle fatigue of diagonally cracked reinforced concrete bridge girders: field tests," Journal of Bridge Engineering, vol. 11, no. 6, pp. 699-706, 2006.

[3] G. Magnel, Theorie und Praxis des Spann-Betons, Bauverlag, 3rd edition, 1956.

[4] T. R. Overman and J. E. Breen, "Fatiguebehavior of pretensioned concretegirders," Tech. Rep., University of Texas, Austin, Tex, USA, 1984.

[5] C. Rao and G. C. Frantz, "Fatigue tests of 27-year-old prestressed concrete bridge box beams," PCI Journal, vol. 41, no. 5, pp. 7483, 1996.

[6] A. Carpinteri, A. Spagnoli, and S. Vantadori, "Mechanical damage of ordinary or prestressed reinforced concrete beams under cyclic bending," Engineering Fracture Mechanics, vol. 72, no. 9, pp. 1313-1328, 2005.

[7] G. Heeke, Untersuchung zur Ermüdungsfestigkeit von Betonstahl undSpannstahl im Zeit- und Dauerhaftigkeits-bereich mit sehr hohen Lastwechselzahlen [Ph.D. thesis], Technical University of Dortmund, Dortmund, Germany, 2016.

[8] J. K. Patel, "Prediction intervals-a review," Communications in Statistics: Theory and Methods, vol. 18, no. 7, pp. 2393-2465, 1989.

[9] W. Q. Meeker and L. A. Escobar, Statistical Methods for Reliability Data, John Wiley \& Sons, New York, NY, USA, 1998.

[10] H. Goual and N. Seddik-Ameur, "Chi-squared type test for the AFT-generalized inverse Weibull distribution," Communications in Statistics-Theory and Methods, vol. 43, no. 13, pp. 2605-2617, 2014.

[11] W. Q. Meeker, L. A. Escobar, and C. J. Lu, "Accelerated degradation tests: modeling and analysis," Technometrics, vol. 40, no. 2, pp. 89-99, 1998.

[12] V. R. B. De Oliveira and E. A. Colosimo, "Comparison of methods to estimate the time-to-failure distribution in degradation tests," Quality and Reliability Engineering International, vol. 20, no. 4, pp. 363-373, 2004.

[13] X. Wang and D. Xu, "An inverse Gaussian process model for degradation data," Technometrics, vol. 52, no. 2, pp. 188-197, 2010.

[14] D. L. Snyder and M. I. Miller, Random Point Processes in Time and Space, Springer, New York, NY, USA, 2nd edition, 1991.

[15] K. Sobczyk and J. Spencer, Random Fatigue. From Data to Theory, Academic Press, Boston, Mass, USA, 1992.

[16] M. Sánchez-Silva and G.-A. Klutke, Reliability and Life-Cycle Analysis of Deteriorating Systems, Springer Series in Reliability Engineering, Springer, Heidelberg, Germany, 2016.

[17] E. Cramer and U. Kamps, "Sequential order statistics and $\kappa$-outof- $n$ systems with sequentially adjusted failure rates," Annals of the Institute of Statistical Mathematics, vol. 48, no. 3, pp. 535549, 1996.

[18] M. Burkschat, "Systems with failure-dependent lifetimes of components," Journal of Applied Probability, vol. 46, no. 4, pp. 1052-1072, 2009.

[19] E. A. Peña, E. H. Slate, and J. R. González, "Semiparametric inference for a general class of models for recurrent events," Journal of Statistical Planning and Inference, vol. 137, no. 6, pp. 1727-1747, 2007. 
[20] K. W. Fertig, M. E. Meyer, and N. R. Mann, "On constructing prediction intervals for samples from a Weibull or extreme value distribution," Technometrics, vol. 22, no. 4, pp. 567-573, 1980.

[21] M. Engelhardt and L. J. Bain, "Prediction intervals for the weibull process," Technometrics, vol. 20, no. 2, pp. 167-169, 1978.

[22] M. Engelhardt and L. J. Bain, “On prediction limits for samples from a weibull or extreme-value distribution," Technometrics, vol. 24, no. 2, pp. 147-150, 1982.

[23] J. Frey, "Prediction bands for the EDF of a future sample," Journal of Statistical Planning and Inference, vol. 142, no. 2, pp. 506-515, 2012.

[24] J. F. Lawless and M. Fredette, "Frequentist prediction intervals and predictive distributions," Biometrika, vol. 92, no. 3, pp. 529$542,2005$.

[25] K. Krishnamoorthy, Y. Lin, and Y. Xia, "Confidence limits and prediction limits for a Weibull distribution based on the generalized variable approach," Journal of Statistical Planning and Inference, vol. 139, no. 8, pp. 2675-2684, 2009.

[26] C. Xiong and G. A. Milliken, "Prediction for exponential lifetimes based on step-stress testing," Communications in Statistics-Simulation and Computation, vol. 31, no. 4, pp. 539556, 2002.

[27] R. Maurer, G. Heeke, and G. Marzahn, "Fatigue strength of prestressing steel tendons embedded in concrete of an aged highway bridge/Ermüdungsfestigkeit derSpannstähle einer Autobahnbrücke von 1957 im einbetonierten Zustand," Bauingenieur, vol. 87, no. 5, pp. 226-236, 2012.

[28] O. Basquin, "The exponential law of endurance tests," Tech. Rep., ASTM, Philadelphia, Pa, USA, 1910.

[29] S. M. Ross, Introduction to Probabilitymodels, Elsevier/ Academic Press, Amsterdam, The Netherlands, 11th edition, 2014.

[30] I. Gertsbakh, E. Neuman, and R. Vaisman, "Monte Carlo for estimating exponential convolution," Communications in Statistics. Simulation and Computation, vol. 44, no. 10, pp. 26962704, 2015.

[31] Z. I. Botev, P. L’Ecuyer, G. Rubino, R. Simard, and B. Tuffin, "Static network reliability estimation via generalized splitting," INFORMS Journal on Computing, vol. 25, no. 1, pp. 56-71, 2013.

[32] A. M. Mood, F. A. Graybill, and D. C. Boes, Introduction to the Theory of Statistics, McGraw-Hill, New York, NY, USA, 3rd edition, 1974. 

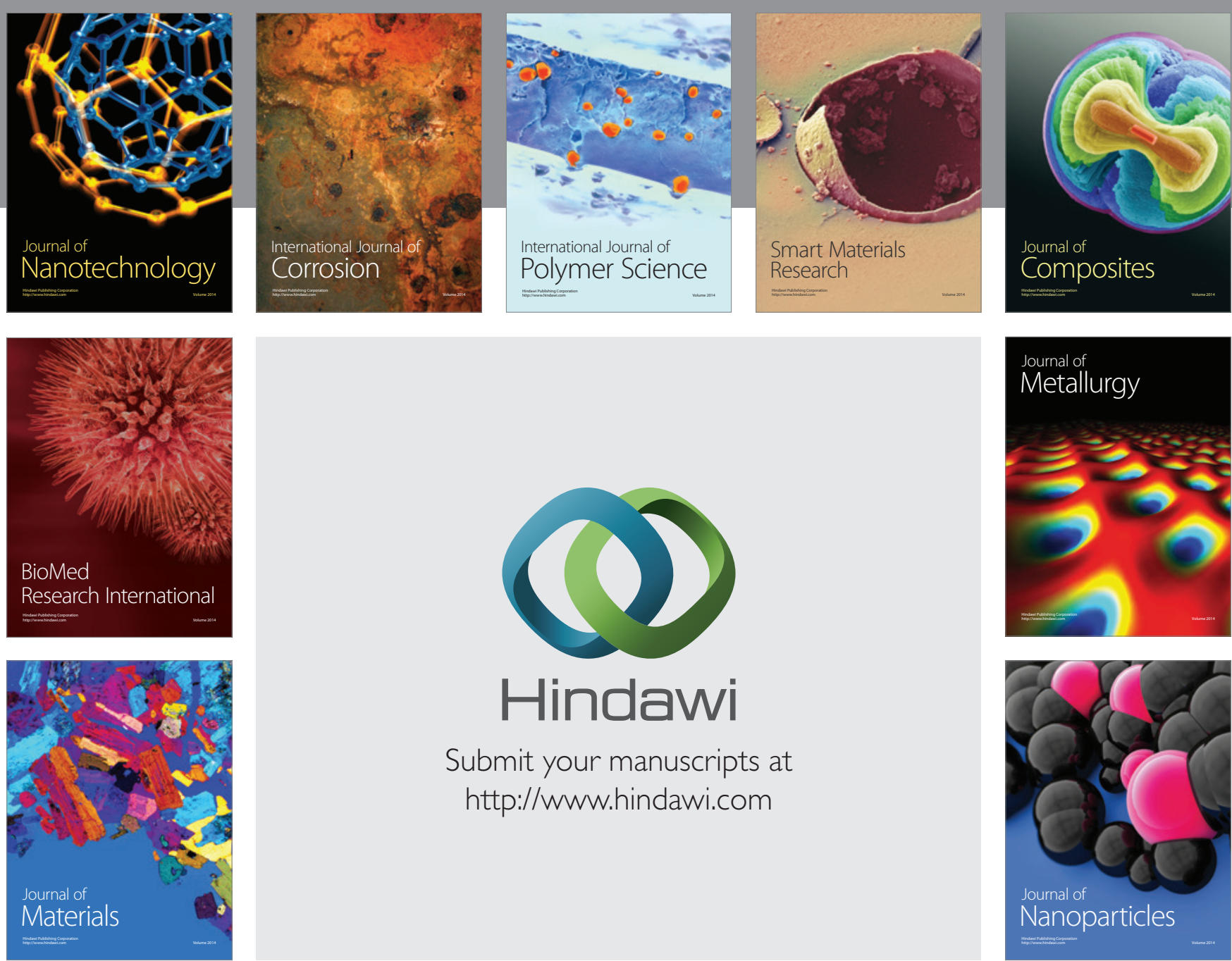

\section{Hindawi}

Submit your manuscripts at

http://www.hindawi.com

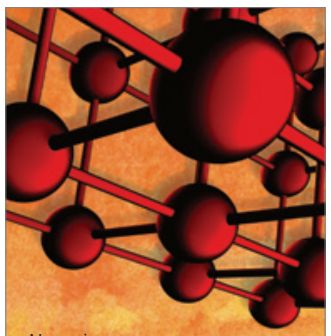

Materials Science and Engineering
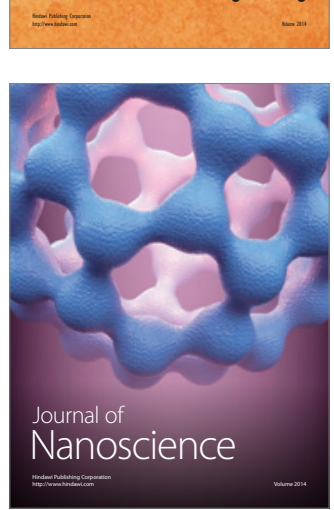
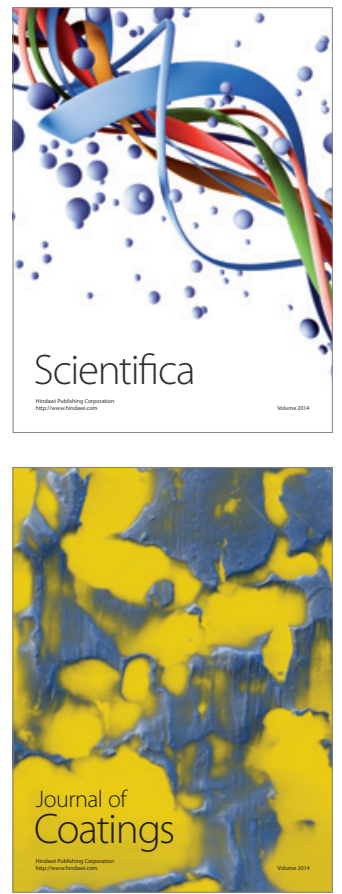
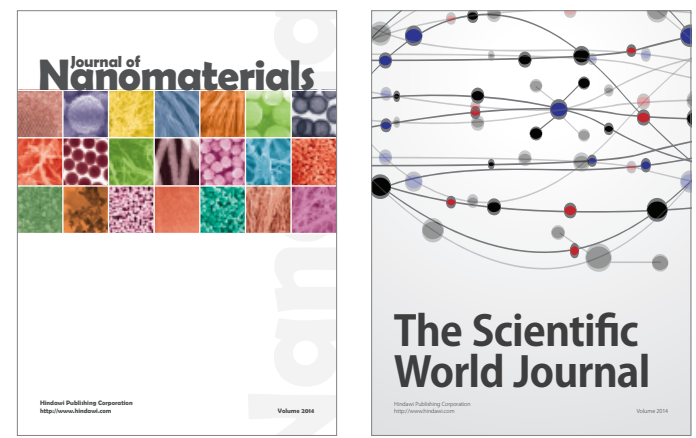

The Scientific World Journal
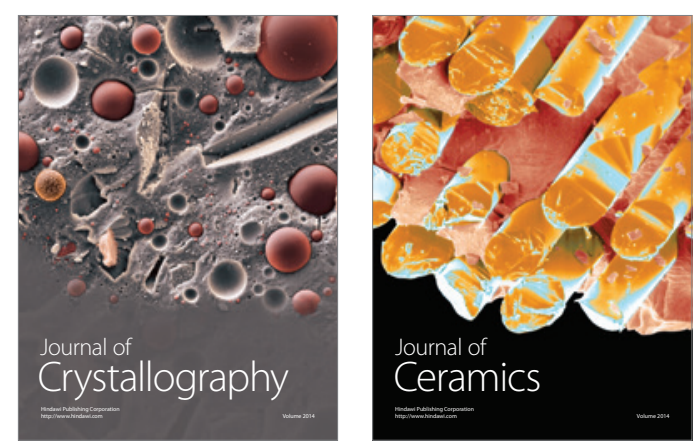
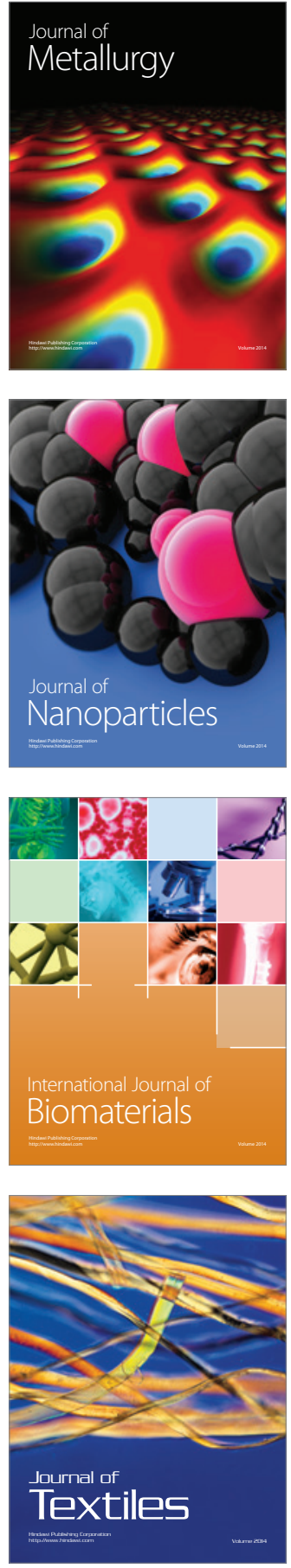